\title{
Heterogeneous Response of Chemotherapy-Related Cognitive Decline in Patients with Breast Cancer: A Prospective Study
}

\author{
Wim Schrauwen ${ }^{1} *$ (D) , Joris Van de Cavey $^{2}$, Guy Vingerhoets ${ }^{3}$, Stijn Vanheule ${ }^{4}$, Rudy Van den Broecke and Hannelore Denys $^{6}$ \\ ${ }^{1}$ Department of Medical Psychology, Ghent University Hospital, Ghent, Belgium \\ ${ }^{2}$ Karus VZW, Campus Melle, in partnership with Ghent University Hospital, Ghent, Belgium \\ ${ }^{3}$ Department of Experimental Psychology, Ghent University, Ghent, Belgium \\ ${ }^{4}$ Department of Psychoanalysis and Clinical Consulting, Ghent University, Ghent, Belgium \\ ${ }^{5}$ Department of Gynaecology, Ghent University Hospital, Ghent, Belgium \\ ${ }^{6}$ Department of Medical Oncology, Ghent University Hospital, Ghent, Belgium
}

(Received June 27, 2019; Final Revision January 7, 2020; AcCEPTEd February 21, 2020)

\begin{abstract}
Objective: A significant proportion of adjuvant-treated breast cancer patients experience cognitive decline, challenging the person's ability to return to normal activities after treatment. However, not every patient experiences cognitive problems, and even in patients with impairments, determining clinically important cognitive decline remains challenging. Our objective was to explore differences in neuropsychological performance following adjuvant chemotherapy (CT) in patients with breast cancer. Method: We conducted a prospective observational study in an Oncology Breast Clinic and assessed neuropsychological performance before and after adjuvant CT and in non-CTtreated women with breast cancer and healthy controls (HCs). Standardised between-group differences and regressionbased change scores were calculated. Results: CT-treated patients $(n=66)$ performed significantly different from non-CT-treated patients $(n=39)$ and HCs $(n=56)$. There was a significant effect on verbal fluency $(p=.0013)$. CT performed significantly worse than non-CT and HC [effect size $(\mathrm{ES})=.89, p<.001$ and $\mathrm{ES}=.61, p \leq .001$, respectively] and from HCs with regard to proactive interference $(\mathrm{ES}=.62, p \leq .001)$. Regression-based scores revealed more severe cognitive decline in the CT-treated group [24.24\% (16/66)] than in the non-CT-treated group [15.20\% (6/39)] and HC group [7.14\% (4/56)]. Patients who underwent CT and showed cognitive decline were less educated and older, with significantly lower baseline scores. Conclusions: CT-treated patients showed more vulnerability on cognitive control and monitoring than non-CT-treated breast cancer patients and HCs. Older patients with less education and lower baseline cognitive performance represent a group at risk for cognitive decline following CT. Identification of patients at risk for decline could improve targeted support and rehabilitation.
\end{abstract}

Keywords: Breast cancer, Chemotherapy, Cognitive dysfunction, Neuropsychology, Executive function, Memory

\section{INTRODUCTION}

Breast cancer is the most common cancer in women worldwide with 201 million newly diagnosed cases in 2018 (Bray et al., 2018). Improved treatment results in an approximately $90 \%$ disease-free 5 -year survival rate, with a sustained rise in long-term survival rates (Noone et al., 2018). However, patients are often left with a cluster of symptoms that negatively affect their quality of life (Mitchell, 2007; Mols, Vingerhoets, Coebergh, \& van de Poll-Franse, 2005), thereby influencing their abilities to regain everyday

*Correspondence and reprint requests to: Wim Schrauwen, Department of Medical Psychology, Ghent University Hospital, Corneel Heymanslaan 10, 9000 Ghent, Belgium. E-mail: wim.schrauwen@uzgent.be function and resume vocational and social activities (De Vries, Den Oudsten, Jacobs, \& Roukema, 2014).

Recently, the gradual shift towards patient-reported outcomes has led to the examination of the impact of cancer and its treatment on cognitive function, one of the most common concerns raised by breast cancer survivors, and an aspect of everyday function that is critical to normal performance. The term 'chemobrain' expresses the burden experienced by long-term survivors (Schagen et al., 2014).

A considerable number of neuropsychological studies, supported by animal models and neuroimaging research, suggest cognitive changes in breast cancer survivors, especially following chemotherapy (CT) (Pomykala, de Ruiter, Deprez, McDonald, \& Silverman, 2013; Seigers, Schagen, Van Tellingen, \& Dietrich, 2013). Although CT is known to be 
cytotoxic to the central nervous system (CNS), prior research showed that 'chemobrain' is a complex phenomenon involving various factors. Besides chemo-induced toxicity, hormonal changes and inflammatory processes are likely to contribute to the development of cognitive problems (Ahles \& Saykin, 2007; Nelson \& Suls, 2013; Scherling et al., 2011; Wefel \& Schagen, 2012). Also, psychological distress and fatigue could theoretically result in impaired neuroplasticity and impaired cognitive performance (Andreotti, Root, Ahles, McEwen, \& Compas, 2015; Hermelink, 2015). Various candidate mechanisms and a spectrum of symptoms should be considered when cancer-associated cognitive decline is investigated (Ahles \& Saykin, 2007; Ahles \& Hurria, 2018; Andreotti et al., 2015; Hermelink, 2015; Wefel \& Schagen, 2012).

Cognitive domains most commonly affected in breast cancer patients are attention and processing speed, memory, and executive function. The latter includes working memory, cognitive flexibility, and multitasking (Ahles et al., 2010; Wefel, Saleeba, Buzdar, \& Meyers, 2010). Root, Andreotti, Tsu, Ellmore, and Ahles (2016) argued that memory is not affected per se, but that an inefficiency in learning is wrongly perceived by patients as forgetting, possibly due to inefficiency of attentional processes. Chen, $\mathrm{Xu}$, $\mathrm{Li}$, Tang, and Wang (2016) showed that following CT, patients had selective abnormality in alerting and executive control networks. These findings should be considered when selecting the cognitive domains to measure in neuropsychological assessment.

There are two major challenges in translating clinically observed symptoms to standardised measures of cognitive impairment. Firstly, various methods are used to define cognitive decline after cancer at the individual level. Most studies define impairment as a clinically relevant difference when comparing patient performance to normative data or performances of non-CT-treated patients or healthy controls (HCs). The boundary between normal and impaired function depends upon arbitrary thresholds, as it relates to both patient performance and the normative standard to which it is compared (Schilder et al., 2010; Shilling, Jenkins, \& Trapala, 2006). Therefore, it may be interesting to focus on patient performance before therapy, rather than a normative score, since even in affected patients, post-treatment scores usually stay within normal boundaries (Jenkins et al., 2006). Moreover, the degree and nature of cognitive impairment after cancer are usually assessed in terms of statistical differences in neuropsychological performance between treatment groups. By estimating differences in performance, a statistical threshold of significance relates to clinical differences and sample characteristics, such as group size. Therefore, by comparing performance between groups, statistical significance should be accompanied by a normalisation of scores that are typically observed in the population to provide information on the severity and impact of cognitive burden (Bernstein, McCreath, Komeylian, \& Rich, 2017; Menning et al., 2016).

Secondly, not all patients following the same treatment trajectory experience cognitive decline to the same extent, or even at all. Some patients might even show cognitive 'improvement', a common finding after repeated assessments, known as the test-retest effect. Accordingly, average group scores may appear stable over time, while individual cases may show marked differences. Thus, even if no mean difference between treatment groups can be found, it remains important to examine the possibility of a vulnerable subpopulation of 'at-risk' patients (Schagen, Muller, Boogerd, Mellenbergh, \& van Dam, 2006), which requires further exploration of individual risk factors to optimise rehabilitation programs.

Our goal was to investigate the prevalence of declined performance on neuropsychological tests grouped into the domains of executive function, verbal memory, and processing speed before and immediately after $\mathrm{CT}$ for breast cancer, compared to performance in non-CT-treated patients and HCs. We focused on the effect sizes (ESs) of between-group differences in performance change between baseline and post-treatment measurements and considered the possibility of a vulnerable sub-population with a regression-based model to identify patients with significant cognitive decline.

\section{METHOD}

\section{Participants}

Patients were recruited at Ghent University Hospital Breast Clinic between August 2010 and December 2012. All patients were between 25 and 65 years of age with newly diagnosed carcinoma of the breast. They were selected for adjuvant treatment after surgery. One patient group received CT [5-fluorouracil, epirubicin, and cyclophosphamide (FEC)] every 21 days for three cycles, followed by three cycles of docetaxel (D) CT (CT group), and, if necessary, radiotherapy (RT) and/or endocrine therapy (ET). The other patient group received RT and/or ET only (non-CT group). The HC group was recruited from age-matched family and friends of participants, and women who responded to the invitation to participate on the website of the hospital. Eligibility screening excluded women with any history of cancer diagnoses and treatment, and with psychiatric or neurological disorders, as well as those not fluent in Dutch. Potential eligible patients were approached by their healthcare providers. The first author provided information and obtained informed consent. Of the 196 patients eligible, 111 agreed to participate. The main reason for refusal was that participation was considered too burdensome. One patient was excluded because of an inability to comprehend task instructions (Figure 1).

All participants provided written informed consent. The Ethical Committee of Ghent University Hospital approved the protocol (ref: B67020108507).

\section{Procedure}

After surgery, but before the start of adjuvant treatment (T1), each participant completed a standardised neuropsychological battery of approximately 1 hour in a set order. For the CT 


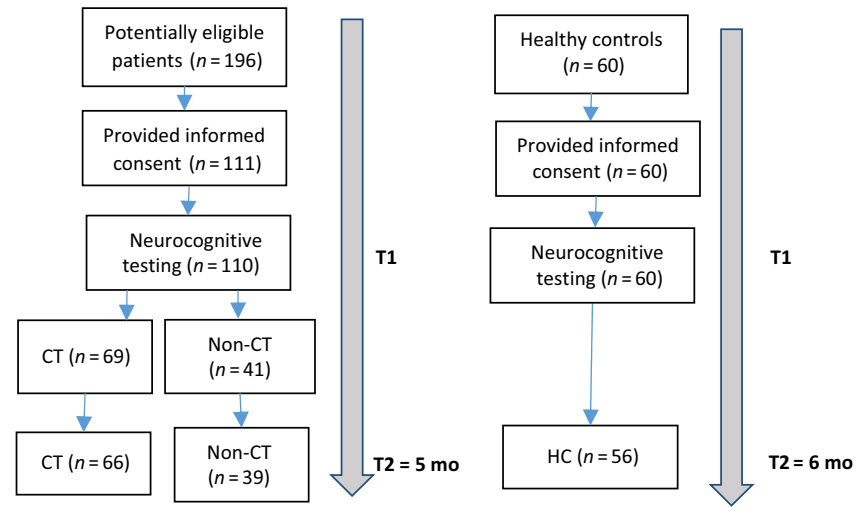

Fig. 1. Flowchart of participants. CT, chemotherapy; mo, months.

group, a second assessment (T2) was performed after the last cycle of CT, but before the start of RT and/or ET. The non-CT group was tested within the same time frame, before the start of adjuvant therapy and 5 months later. The HC group was tested and retested with a 6-month time interval. A standardised neuropsychological battery of approximately 1 hour was administered to each patient in a set order.

\section{Neuropsychological Measures}

\section{Verbale Leer-en Geheugen Test}

The Dutch version of the California Verbal Learning Test (Verbale Leer-en Geheugen Test - VLGT) is a measure of verbal memory commonly used to assess both learning and memory. The presence of a second list B in the VLGT allows measurement of the effects proactive and retroactive interference (RI) on learning and memory (Wohldmann, Healy, \& Bourne, 2008). We retained the total score of list A after five recall attempts, the distraction list $\mathrm{B}$, the immediate recall of $\mathrm{A}$, and the delayed recall after 20 minutes, together with proactive interference (PI) and RI.

\section{D-KEFS Colour-Word Test}

The D-KEFS Colour-Word Test (Delis, Kaplan, \& Kramer, 2001) is designed to measure executive control of attention by involvement of interference of prepotent responses. The test consists of four trials, the first two consisting of word reading and colour naming and measuring speed of information processing. The third and fourth trials are executive function tasks.

\section{D-KEFS Trail Making Test}

The Trail Making Test of the Delis-Kaplan Executive Function System (Delis et al., 2001) assesses set-shifting: the ability to fluently switch between different mental sets. We included all variables: visual scanning, number sequencing, letter sequencing as speed of information processing of the different component skills of this task, and executive function number-letter switching.

\section{Controlled Oral Word Association Test}

Verbal fluency was assessed with the Controlled Oral Word Association Test (Benton, Hamsher, \& Sivan, 1994), a measure of phonemic and semantic fluency (Miatton, Lannoo, \& Vingerhoets, 2004).

Finally, a Dutch version of the National Adult Reading Test (NART [Nelson \& Willison, 1991]; DART [Schmand, Lindeboom, \& Harskamp, 1992]) was administered to obtain an estimation of intelligence.

\section{Statistical Analysis}

SPSS Statistics for Windows (version 25.0, IBM Corp., Armonk, NY, USA) was used for descriptive analyses. All other analyses were performed in $\mathrm{R}$ (Version 3.0.2, $\mathrm{R}$ Foundation for Statistical Computing, Vienna, Austria).

\section{Standardisation of the data set}

For interpretation purposes, raw scores of neuropsychological tests were converted into standardised scores based on the mean and standard deviation (SD) of the HC group at T1. Hence, for the HC group at T1, each parameter was distributed with zero mean and SD equal to 1.

\section{Group differences}

T2 scores were also standardised based on the mean and the $\mathrm{SD}$ of the HC group on T1. This allowed us to then calculate differences between $\mathrm{T} 2$ and $\mathrm{T} 1$ scores which were based on the same standardisation, thus providing a more direct link with raw differences (as opposed to, for example, T2 corrected for T1). Paired samples t-tests were conducted to compare performance in all neuropsychological measures between HCs and the two patient groups. ESs were calculated as Cohen's d (mean paired differences/SD paired differences). We calculated the difference score (T2 minus T1) and assessed significant differences between the groups. P-values indicated group as a significant predictor. Applying multi-comparison Bonferroni correction, we deemed differences with $p<.001$ as statistically significant. For each test value, groups were compared, and we calculated ES, confidence interval (CI), and p-value. Generally, an ES of .8 or more is considered a large effect, an ES of .5 is considered moderate, and an ES of .2 is considered a small effect.

\section{Regression-Based Change}

As previously mentioned, it is important to compare group differences and to determine which subgroups of patients show relevant decline. To detect which subgroup of patients show clinically relevant decline, we used RBC scores (McSweeny, Naugle, Chelune, \& Lüders, 1993), which originate from differences in initial baseline scores, considering relevant demographic variables that could influence change in a test-retest situation.

For the different test variables, we calculated optimal regression coefficients by regressing the retest scores for 
the HC group against their prior scores (Supplementary Material A). Potential contributing factors, such as age, intelligence, education, and fatigue, were added to the equation, together with sleep quality and menopausal status (Henneghan, Carter, Stuifbergan, Parmelee, \& Kesler, 2018; Lord, Buss, Lupien, \& Pruessner, 2008). Beginning with performance on $\mathrm{T} 1$, the predicting variables were incrementally added until the best model was achieved. T1 score and age were important contributing factors. Following these regression models (Supplementary Material B), we calculated optimal regression coefficients and predicted $\mathrm{T} 2$ scores for patients in the CT and non-CT groups.

To determine whether a patient's retest performance significantly deviated from normal expectations, we compared the observed retest score with the regression-predicted retest score and divided the difference by the mean square error. We obtained standardised difference scores that represent to what extent the actual score on T2 deviated from what would be expected based on the T1 score and the change occurring in the $\mathrm{HC}$ group. The standardisation of the T2 scores based on the mean and SD of HCs at T1 distributes solely for ability ( + error). However, we argue that degree of change expected in controls is modelled for using the linear regression models. These are oriented towards estimating how much a patient in the control group, given a certain starting ability and demographic factors, should be expected to show practice effects. By contrasting the standardised T2 scores of the experimental conditions against the expected scores based on these models, we thus investigate these groups relative to both ability and practice effects in the control group.

To delineate which participants deviated significantly from their expected T2 scores (and can thus be said to have experienced cognitive decline), it is necessary to set a cut-off point to the RBC scores. The heterogeneity of definitions of impairment makes such conclusions somewhat arbitrary. Given the large differences that exist in the determination of a threshold in previous papers (Schilder et al., 2010), we have decided to include a table detailing the prevalence of 'declined' subgroups in the different conditions for several thresholds. Even though we decided to uphold the standard of choosing $2.5 \mathrm{SD}$, we agree that placing the boundary at $-2.5 \mathrm{SD}$ is a subjective decision (which is why we chose to also include what the 'declined' group would look like following $-1.5 \mathrm{SD}$ and $-2 \mathrm{SD})$. We applied the reasoning that if the 'significantly worse performing' group was based on a boundary less than $-2.5 \mathrm{SD}$, this would not follow the restrictions typically placed on determining outliers within a distribution. It might be relevant for other papers to better compare these subgroups to their own threshold selection (Schagen et al., 2006).

\section{RESULTS}

\section{Demographic and Clinical Characteristics}

Demographic and clinical characteristics of both patient groups and HCs are presented in Table 1. From the final sample of 110 patients that completed T1, 69 received adjuvant $\mathrm{CT}$ (CT group). Forty-one patients received RT and/or ET (non-CT group). Ninety-six percent of patients in the CT group received the combination of FEC/D. All but one patient $(97.6 \%)$ in the non-CT group received adjuvant ET, compared to $77 \%$ in the CT group. The average age of all women was 49.1 years. Patients in the non-CT group were significantly older, and adjuvant CT was associated with higher cancer stage. Nearly $90 \%$ of patients in the non-CT group had a pT1 tumour, while $50.7 \%$ of those in the CT group had pT2 breast cancer. Thirty-nine patients in the CT group (56.4\%) and $19.5 \%$ in the non-CT group had positive lymph nodes. Before treatment, $35 \%, 54 \%$, and $43 \%$ of patients in the $\mathrm{CT}$, non-CT, and $\mathrm{HC}$ groups, respectively, were postmenopausal. No significant differences in education and estimated intelligence quotient (IQ) were found. Three patients in the CT group and two in the non-CT group dropped out at T2. In the HC group, 4 of 60 participants dropped out by $\mathrm{T} 2$. Thus, complete data were obtained from 105 patients and $56 \mathrm{HCs}$.

\section{Group Differences in Cognitive Change}

Patient groups and controls did not differ in most neuropsychological tests (Supplementary Materials C and D), but a significant difference was found for verbal fluencyletters $(p=.0013)$, with a large ES between the CT and non-CT groups $(\mathrm{ES}=.89, p \leq .001)$ and moderate ESs on verbal fluency-letters between the $\mathrm{CT}$ and $\mathrm{HC}$ groups ( $\mathrm{ES}=.61, \mathrm{p}=.0011)$ and for VLGT PI between the CT and $\mathrm{HC}$ groups $(\mathrm{ES}=.62, p \leq .001)$. Thus, the $\mathrm{CT}$ group showed significant decline on letter fluency and the PI task of the VLGT compared to the other groups.

\section{Cognitive decline versus no cognitive decline}

As explained earlier, we systematically selected subgroups of patients with standardised difference scores of $-2.5 \mathrm{SD}$. Using the -2.5 points threshold, the percentages of subjects with cognitive decline were $24.24 \%$ (16/66) in the CT group, $15.20 \%(6 / 39)$ in the non-CT group, and $7.14 \%(4 / 56)$ in the HC group (Supplementary Material E). Therefore, the proportion of participants performing lower than expected between $\mathrm{T} 1$ and $\mathrm{T} 2$ significantly differed between the groups $\left(\chi^{2}=6.973, p=.030608\right)$.

Furthermore, to link this relative decline in cognitive performance to actual differences in neuropsychological measures, we used independent group comparisons for raw scores at T2; observing that the subgroup of participants with cognitive decline had significantly lower raw scores at $\mathrm{T} 2$ than those without cognitive decline, especially in the CT group (Supplementary Material F). For all analyses, a $p$-value $<.01$ was considered statistically significant. The CT group with cognitive decline scored significantly lower than the group without cognitive decline on the VLGT total recall $(p=.0007)$, short delay recall $(p=.0029)$, long delay recall 
Table 1. Disease and demographic characteristics of the sample

\begin{tabular}{|c|c|c|c|c|c|c|c|c|}
\hline \multirow[b]{2}{*}{ Characteristics } & \multicolumn{2}{|c|}{$\begin{array}{l}\text { Patients scheduled for } \\
\text { chemotherapy }(n=69)\end{array}$} & \multicolumn{2}{|c|}{$\begin{array}{l}\text { Patients scheduled for } \\
\text { non-chemotherapy } \\
\text { treatment }(n=41)\end{array}$} & \multicolumn{2}{|c|}{$\begin{array}{l}\text { Healthy controls } \\
\quad(n=56)\end{array}$} & \multicolumn{2}{|c|}{$\begin{array}{c}\text { One-way ANOVA } \\
\text { or Mann-Whitney } \\
\text { U-test }\end{array}$} \\
\hline & Mean or count & SD & Mean or count & $\mathrm{SD}$ & Mean or count & SD & F-statistic & $p$ \\
\hline Age at baseline years (range) & $46.7(27-64)$ & 9.7 & $53.1(44-65)$ & 5.6 & $47.9(25-64)$ & 10.6 & 7.643 & .001 \\
\hline pTNM classification & & & & & & & & $<.001 *$ \\
\hline $\mathrm{T} 1$ & $21(30.4 \%)$ & & $36(87.8 \%)$ & & NA & & & \\
\hline $\mathrm{T} 2$ & $35(50.7 \%)$ & & $5(12.2 \%)$ & & NA & & & \\
\hline $\mathrm{T} 3$ & $12(17.4 \%)$ & & NA & & NA & & & \\
\hline $\mathrm{T} 4$ & $1(1.4 \%)$ & & NA & & NA & & & \\
\hline Positive lymph nodes & $39(56.4 \%)$ & & $8(17.1 \%)$ & & NA & & & $<.001 *$ \\
\hline Menopausal status & & & & & & & .497 & .609 \\
\hline Premenopausal & $45(65.2)$ & & $19(46.3 \%)$ & & $32(57.1 \%)$ & & & \\
\hline Postmenopausal & $24(34.8)$ & & $22(53.7 \%)$ & & $22(42.9 \%)$ & & & \\
\hline Surgical treatment & & & & & & & & $.001 *$ \\
\hline Lumpectomy & $30(43.5 \%)$ & & $33(80.5 \%)$ & & NA & & & \\
\hline Mastectomy & $35(50.6 \%)$ & & $8(19.5 \%)$ & & NA & & & \\
\hline \multicolumn{9}{|l|}{ Radiotherapy } \\
\hline $\mathrm{y}$ & $65(94.2 \%)$ & & $36(87.8 \%)$ & & NA & & & .239 \\
\hline $\mathrm{n}$ & $4(5.8 \%)$ & & $5(12.2 \%)$ & & & & & \\
\hline \multicolumn{9}{|l|}{ Hormonal therapy } \\
\hline $\mathrm{y}$ & $53(76.8 \%)$ & & $40(97.6 \%)$ & & NA & & & $.004^{*}$ \\
\hline $\mathrm{n}$ & $16(23.2 \%)$ & & $1(2.4 \%)$ & & & & & \\
\hline Education years & 13.7 & 2.9 & 13.5 & 2.6 & 14.5 & 2 & 1.968 & .114 \\
\hline Estimated IQ & 111.4 & 10.2 & 112.8 & 11.4 & 116.5 & 10.2 & 1.492 & .228 \\
\hline
\end{tabular}

ANOVA, analysis of variance; IQ, intelligence quotient; NA, not applicable; SD, standard deviation; pTNM, classification of malignant tumours; y-n: yes - no. *Disease variables between the two patient groups were assessed with the Mann-Whitney U Test; significance values are reported.

$(p=.009)$, D-KEFS visual scanning $(p=.002)$, D-KEFS letter $(p=.0022)$, D-KEFS switching $(p=.001)$, D-KEFS word reading $(p=.003)$, D-KEFS inhibition $(p=.00043)$, D-KEFS inhibition/switching $(p=.0006)$, and verbal fluency-letter ( $p=.0001)$ tests. In the non-CT group, there were only significant differences on verbal fluency-animals $(p=.0065)$ and profession $(p=.0018)$. No statistically significant difference $(p<.01)$ was retained between any measures in HCs.

When relating the raw $\mathrm{T} 2$ scores to the $\mathrm{T} 1$ scores for the groups with and without cognitive decline, results indicated that the group with cognitive decline typically consisted of those who had already lower performance at T1, which remained within the normal range. No statistically significant differences $(p<.001)$ between any measures in the non-CT and $\mathrm{HC}$ groups with and without cognitive decline were retained at $\mathrm{T} 1$. In the CT group, the group that showed 'later decline' performed significantly worse on T1 than the group that did not show later decline in the VLGT total recall $(p=.0015)$, short delay recall $(p=.0088)$, D-KEFS TM numbers $(p=.0033)$ D-KEFS TM letter $(p=.0029)$, and D-KEFS TM switching $(p=.0058)$ tests. Figure 2 depicts the subtle deterioration of the subgroup already scoring lower at $\mathrm{T} 1$.

Finally, patients with and without cognitive decline differed in several features, as summarised in Table 2. Participants with significant cognitive decline had a lower level of education overall, but this was only significant in the CT group. Patients with cognitive decline were also significantly older in the CT group. Participants with cognitive decline showed lower estimated intelligence, but this was only significant in the non-CT group.

\section{DISCUSSION}

The primary focus of this study was to investigate the prevalence of cognitive decline in women with breast cancer immediately after CT, compared to the performance of non-CT treated patients and HCs. Following concerns of previous studies, we attempted to study cognitive decline not through significant deviations from norm scores; however, we focused on ESs in between-group changes using scores standardised based on HC performance. Furthermore, we explored the relevance of discerning an 'at-risk' subgroup, using standardised RBC scores to identify patients with dysfunctional evolutions in their cognitive performance. Thus, we adhered to recent methodological recommendations to evaluate psychometric changes in longitudinal studies (Andreotti et al., 2016).

\section{Group Level}

At the group level, results showed several between-group differences. Both CT and non-CT patient groups showed less resistance to PI at T2. In contrast to the findings by Root et al. (2016), performance in the VLGT distraction list opposing 
Table 2. Comparison of features in patients with and without cognitive decline

\begin{tabular}{llccl}
\hline \hline & Group & Decline & No decline & $p(t$ test $)$ \\
\hline Education years & HC & 13.2 & 14.8 & \\
& Non-CT & 11 & 14 & 0.068 \\
& CT & 10.8 & 14.6 & $0.0006^{*}$ \\
Sleep quality & HC & 7.5 & 7.2 & 0.414 \\
& Non-CT & 7 & 7,1 & 0.732 \\
IQ & CT & 7 & 7 & 0.88 \\
& HC & 112 & 117 & 0.339 \\
Age & Non-CT & 100.5 & 115 & $0.0038^{*}$ \\
& CT & 106 & 113 & 0.089 \\
& HC & 48.2 & 47.9 & 0.9634 \\
Days since surgery & Non-CT & 52 & 53.2 & 0.837 \\
& CT & 53.5 & 44.6 & $0.00037^{*}$ \\
& HC & & & \\
& Non-CT & 20 & 28.5 & 0.0395 \\
& CT & 19.4 & 19.4 & 0.9934 \\
\hline \hline
\end{tabular}

CT, chemotherapy; HC, healthy control; IQ, intelligence quotient. $* p<0.001$. the first encoding of list A seemed more difficult in both groups. The shifting of attention while inhibiting learned material caused problems for patients, whereas HCs showed improvement; therefore, patients were less able to inhibit previously learned - distracting - information. These results are comparable with data published by Andryszak in a recent study on verbal memory in CT-treated breast cancer patients compared to HCs (Andryszak, Wiłkość, Żurawski, \& Izdebski, 2018). The presence of list B did not otherwise inhibit retrieval of previously memorised material (RI). Comparing CT-treated patients with both non-CT and HC groups, we found large ESs for letter fluency [CT vs. NonCT $(\mathrm{ES}=.89)$; CT vs. HC $(\mathrm{ES}=.61)]$. In the letter fluency task, patients must maintain instructions in working memory and suppress semantically related words while adapting novel search strategies; this measures verbal and executive control ability. Prior research suggests the effects of CT on specific domains of executive functioning. These findings suggest that CT-treated patients are vulnerable to cognitive control and monitoring (Chen et al., 2014; Yao, Bernstein, \& Rich, 2017). Deficits in executive functioning may not have

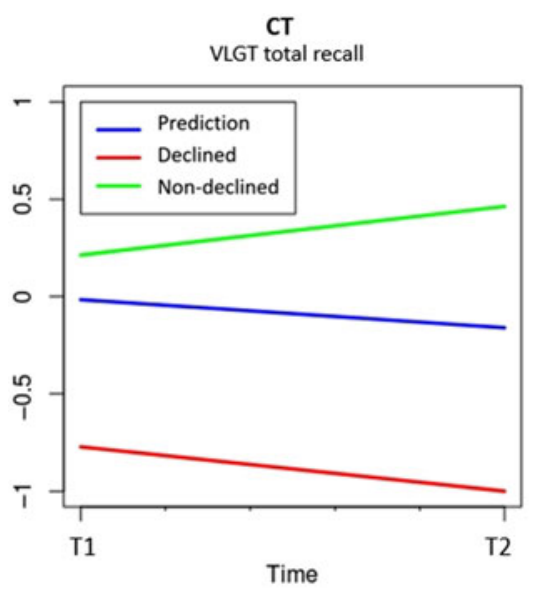

CT
VLGT total recall

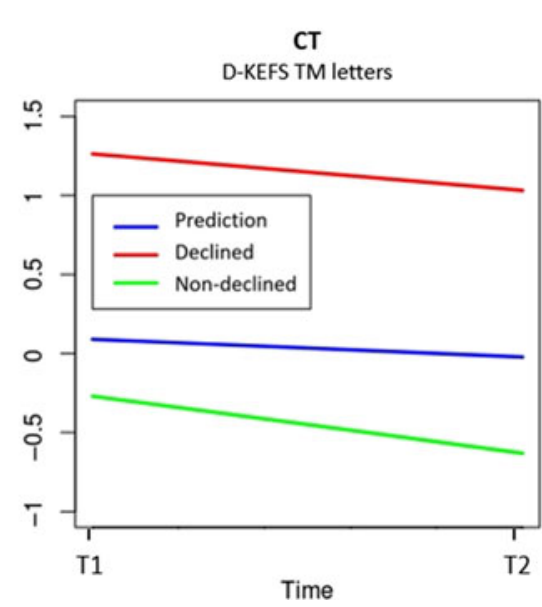

CT

VLGT short delay recall

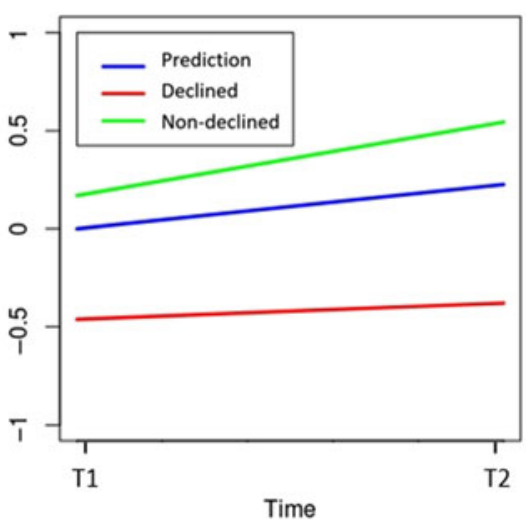

CT

D-KEFS TM numbers

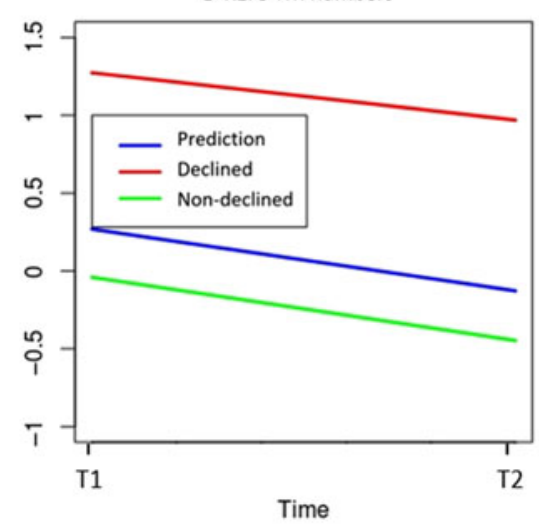

CT

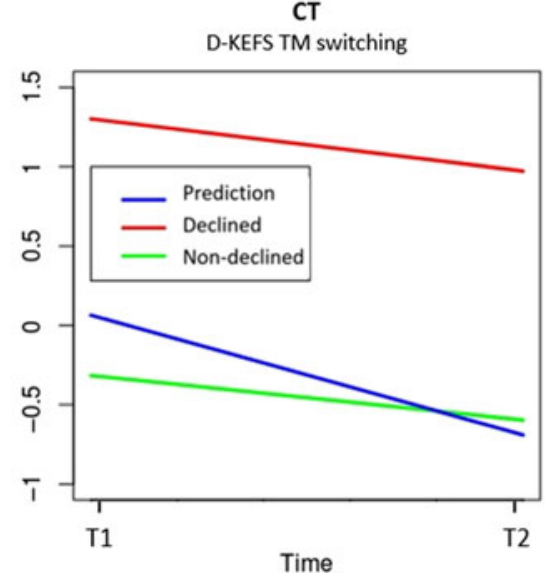

Fig. 2. Visual presentation of declined and non-declined $\mathrm{T} 1$ and $\mathrm{T} 2$. The prediction line consists of the mean of the total $\mathrm{CT}$ group at $\mathrm{T} 1$ and the predicted score at $\mathrm{T} 2$, the latter on the basis of the expected evolution of the CT group, given their basic score, age, education, and other factors in this study, as well as the evolution of the HC group. 
been reflected in the Colour-Word and Trail making task because of a lack of sensitivity in these tests. Moreover, in contrast to these tests, the letter fluency task requires greater effort and a more active strategic search. Regardless of the observation of significant differences on some measures, no other significant group differences were found globally. Despite the reduced performance that was observed in the CT group, the non-CT group also received cancer treatment, such as RT, hormonal therapy, and the additional effects of psychological factors and fatigue (Ahles \& Root, 2018; Andreotti et al., 2015; Boele, Schilder, de Roode, Deijen, \& Schagen, 2015; Menning et al., 2015) with a potential negative impact. Our findings agree with recent meta-analyses that emphasize the subtlety of cognitive dysfunction, but deviate somewhat from the findings that cognitive impairments in breast cancer patients occur independently of CT (Bernstein et al., 2017; Ono et al., 2015).

\section{Individual Level}

We aimed to determine whether a subgroup of patients with cognitive decline could be identified using RBC scores to predict individual performance. We observed a larger subgroup with severe cognitive decline among CT-treated patients, compared to the other groups. Twenty-four percent of patients in the CT group showed scores 2.5 points lower than expected in more than one test at $\mathrm{T} 2$, compared to $15 \%$ and $7 \%$ of those in the non-CT and $\mathrm{HC}$ groups, respectively. Lower education level and older age were significant predictors of cognitive decline in the CT group. An association between decline and age could not be found in the other groups. Other researchers demonstrated associations among cognitive performance, age, and years of education, with a larger effect in CT-treated patients than in non-CT-treated patients (Ahles \& Hurria, 2018; Tager et al., 2010). Dichotomisation of patients into those with and without cognitive decline could have concealed the fact that all CT-treated patients experience negative cognitive effects; however, only patients with lower IQ and education were unable to maintain task performance, whereas younger patients with higher education were more resilient to decline.

\section{Study Limitations}

This study had several limitations. First, a number of patients declined to participate, mainly because of the burden of the given diagnosis at that time. Second, all participants were white and Flemish, the majority of whom were highly educated and financially stable, limiting the external validity of the findings. Given the small number of included participants, a further limitation is that the study may have been underpowered to identify predictors of cognitive decline within subgroups. Fourth, we evaluated the acute, short-term effects of CT; we did not include the analysis of long-term cognitive performance of the patient groups. And finally, in this paper, we did not include an assessment of perceived cognitive decline nor of any other possible contributing psychological factors. Since mechanisms that cause cognitive decline in breast cancer patients are likely to be multifactorial, these factors may increase vulnerability to cognitive deterioration and should be considered for future research.

\section{Clinical Implications}

The results corroborate the hypothesis that $\mathrm{CT}$ is an important factor in cognitive decline in breast cancer patients. The findings of this study can alert healthcare professionals of the need to identify and support breast cancer patients with potential decline, thereby facilitating targeted rehabilitation after treatment for breast cancer. The findings also emphasise that healthcare professionals and researchers should carefully use terms such as 'impairment' and 'dysfunction'. There is a need to agree on a definition of cognitive decline and standardise the methodology for accurate assessment, thus allowing comparison of studies regarding the prevalence and nature of cognitive dysfunction in patients who have undergone treatment for cancer.

\section{Future Studies}

The possibility that psychological factors increase vulnerability for cognitive decline after cancer treatment remains controversial (Vearncombe \& Pachana, 2009). Core executive functions, such as task-switching and inhibition, are essential for task performance, but also serve self-regulatory abilities. Berman et al. (2014) showed that pre-treatment worry was associated with cognitive impairment; other research has linked the lack of cognitive control to a higher tendency to ruminate (De Lissnyder, Koster, Derakshan, \& De Raedt, 2010). Further research is needed to determine whether cognitive function and perseverative cognitions share common factors.

Finally, the tendency to objectify cognitive complaints after cancer resulted in a neglect of subjective experience. Qualitative studies addressing the phenomenology of cognitive difficulties are limited but can only enrich and complement existing research.

To conclude, our findings revealed no significant global difference in performance between CT- and non-CT-treated participants, aside from cognitive control and monitoring frailty in verbal fluency letters and PI performance. Seventy-five percent of patients in the CT group continued to perform normally; however, compared with HCs, a significantly larger subgroup of participants with cognitive decline could be discerned. In particular, those who already performed more weakly before adjuvant treatment and with lower education levels and higher age were prone to further deterioration in performance; they constituted a vulnerable group. We should, however, not disregard the fact that neuropsychological assessment may not be sufficiently sensitive to capture the complexity of cognitive problems in cancer 
survivors and that patient-reported outcomes should be incorporated into such assessments.

\section{ACKNOWLEDGMENTS}

The authors would like to thank the patients and their friends and families for their voluntary participation.

\section{FUNDING}

This project was supported by Kom op tegen Kanker (Stand up to Cancer), the Flemish League Against Cancer, and a grant from AstraZeneca (grant number: 2007-AZ-338).

\section{CONFLICTS OF INTEREST}

The authors have nothing to disclose.

\section{SUPPLEMENTARY MATERIAL}

To view supplementary material for this article, please visit https://doi.org/10.1017/S1355617720000296

\section{REFERENCES}

Ahles, T.A. \& Hurria, A. (2018). New challenges in psychooncology research IV: cognition and cancer: conceptual and methodological issues and future directions. Psycho-Oncology, 27, 3-9.

Ahles, T.A. \& Saykin, A.J. (2007). Candidate mechanisms for chemotherapy-induced cognitive changes. Nature Reviews Cancer, 7, 192-201.

Ahles, T.A., Saykin, A.J., McDonald, B.C., Li, Y., Furstenberg, C.T., Hanscom, B.S., Mulrooney, T.J., Schwartz, G.N., \& Kaufman, P.A. (2010). Longitudinal assessment of cognitive changes associated with adjuvant treatment for breast cancer: impact of age and cognitive reserve. Journal of Clinical Oncology, 28, 4434-4440.

Ahles TA \& Root JC. (2018). Cognitive effects of cancer and cancer treatments. Annual Review of Clinical Psychology;14(1): annurev-clinpsy-050817-084903. doi: 10.1146/annurev-clinpsy050817-084903

Andreotti, C., Root, J.C., Ahles, T.A., McEwen, B.S., \& Compas, B.E. (2015). Cancer, coping, and cognition: a model for the role of stress reactivity in cancer-related cognitive decline. Psycho-Oncology, 24, $617-623$.

Andreotti, C., Root, J.C., Schagen, S.B., McDonald, B.C., Saykin, A.J., Atkinson, T.M., Li, Y., \& Ahles, T.A. (2016). Reliable change in neuropsychological assessment of breast cancer survivors. PsychoOncology, 25, 43-50.

Andryszak, P., Wiłkość, M., Żurawski, B., \& Izdebski, P. (2018). Verbal memory in breast cancer patients treated with chemotherapy with doxorubicin and cyclophosphamide. European Journal of Cancer Care, 27, e12749.

Benton, A., Hamsher, K., \& Sivan, A.B. (1994). Multilingual aphasia examination: manual of instruction. Iowa City: AJA Associates.

Berman, M.G., Askren, M.K., Jung, M., Therrien, B., Peltier, S., Noll, D.C., Zhang, M., Ossher, L., Hayes, D.F., Reuter-Lorenz, P.A., \& Cimprich, B. (2014). Pretreatment worry and neurocognitive responses in women with breast cancer. Health Psychology, 33, 222-231.

Bernstein, L.J., McCreath, G.A., Komeylian, Z., \& Rich, J.B. (2017). Cognitive impairment in breast cancer survivors treated with chemotherapy depends on control group type and cognitive domains assessed: A multilevel meta-analysis. Neuroscience and Biobehavioral Reviews, 83, 417-428.

Boele, F.W., Schilder, C.M.T., de Roode, M-L., Deijen, J.B., \& Schagen, S.B. (2015). Cognitive functioning during long-term tamoxifen treatment in postmenopausal women with breast cancer. Menopause, 22(1):17-25. doi: 10.1097/GME.00000 00000000271

Bray, F., Ferlay, J., Soerjomataram, I., Siegel, R.L., Torre, L.A., \& Jemal, A. (2018). Global cancer statistics 2018: GLOBOCAN estimates of incidence and mortality worldwide for 36 cancers in 185 countries. CA: A Cancer Journal for Clinicians, 68(6), 394-424. doi: 10.3322/caac.21492.

Chen, C., Xu, G.H., Li, Y.H., Tang, W.X., \& Wang, K. (2016). Selective impairment of attention networks during propofol anesthesia after gynecological surgery in middle-aged women. Journal of the Neurological Sciences, 363, 126-131.

Chen, X., Li, J., Ren, J., Hu, X., Zhu, C., Tian, Y., Ma, H., Yu, F., \& Wang, K. (2014). Selective impairment of attention networks in breast cancer patients receiving chemotherapy treatment. PsychoOncology, 23, 1165-1171.

De Lissnyder, E., Koster, E.H.W., Derakshan, N., \& De Raedt, R. (2010). The association between depressive symptoms and executive control impairments in response to emotional and non-emotional information. Cognition and Emotion, 24, 264-280.

De Vries, J., Den Oudsten, B.L., Jacobs, P.M., \& Roukema, J.A. (2014). How breast cancer survivors cope with fear of recurrence: a focus group study. Supportive Care in Cancer, 22, 705-712.

Delis, D., Kaplan, E., \& Kramer, J.H. (2001). Delis-Kaplan Executive Function System (D-KEFS): Examiner's manual. San Antonio, TX: The Psychological Cooperation.

Henneghan, A.M., Carter, P., Stuifbergan, A., Parmelee, B., \& Kesler, S. (2018). Relationships between self-reported sleep quality components and cognitive functioning in breast cancer survivors up to 10 years following chemotherapy. Psycho-Oncology, 27, 1937-1943.

Hermelink, K. (2015). Chemotherapy and cognitive function in breast cancer patients: the so-called chemo brain. Journal of the National Cancer Institute. Monographs, 2015, 67-69.

Jenkins, V., Shilling, V., Deutsch, G., Bloomfield, D., Morris, R., Allan, S., Bishop, H., Hodson, N., Mitra, S., Sadler, G., Shah, E., Stein, R., Whitehead, S., \& Winstanley, J. (2006). A 3-year prospective study of the effects of adjuvant treatments on cognition in women with early stage breast cancer. British Journal of Cancer, 94, 828-834.

Lord, C., Buss, C., Lupien, S.J., \& Pruessner, J.C. (2008). Hippocampal volumes are larger in postmenopausal women using estrogen therapy compared to past users, never users and men: a possible window of opportunity effect. Neurobiology of Aging, 29, 95-101.

McSweeny, A.J., Naugle, R.I., Chelune, G.J., \& Lüders, H. (1993). "T Scores for Change": an illustration of a regression approach to depicting change in clinical neuropsychology. Clinical Neuropsychologist, 7, 300-312.

Menning, S., de Ruiter, M.B., Kieffer, J.M., Agelink van Rentergem, J., Veltman, D.J., Fruijtier, A., Hester, S.A., Oldenburg, M.D., Boven, E., van der Meij, S., Lustig, V., Bos, 
M.E.M., Boogerd, W., Reneman, L., \& Schagen, S.B. (2016). Cognitive impairment in a subset of breast cancer patients after systemic therapy - results from a longitudinal study. Journal of Pain and Symptom Management, 52, 560-569.e1.

Menning, S., de Ruiter, M.B., Veltman, D.J., Koppelmans, V., Kirschbaum, C., Boogerd, W., Reneman, L., \& Schagen, S.B. (2015). Multimodal MRI and cognitive function in patients with breast cancer prior to adjuvant treatment - the role of fatigue. NeuroImage: Clinical, 7:547-554. doi: 10.1016/j.nicl.2015. 02.005

Miatton, M.W.M., Lannoo, E., \& Vingerhoets, G. (2004). Updated and extended Flemish normative data of commonly used neuropsychological tests. Psychologica Belgica, 44, 189-216.

Mitchell, T. (2007). The social and emotional toll of chemotherapy patients' perspectives. European Journal of Cancer Care, 16, 39-47.

Mols, F., Vingerhoets, A.J., Coebergh, J.W., \& van de Poll-Franse, L.V. (2005). Quality of life among long-term breast cancer survivors: a systematic review. European Journal of Cancer (Oxford, England), 41, 2613-2619.

Nelson, H. \& Willison, J. (1991). National Adult Reading Test Manual (2nd ed.). Windsor: NFER-Nelson.

Nelson, W.L. \& Suls, J. (2013) New approaches to understand cognitive changes associated with chemotherapy for non-central nervous system tumors. Journal of Pain and Symptom Management, 46(5), 707-721.

Noone, A.M., Howlader, N., Krapcho, M., Miller, D., Brest, A., Yu, M., Ruhl, L., Tatalovich, Z., Mariotto, A., Lewis, D.R., Chen, H.S., Feuer, E.J., \& Cronin, K.A. (eds). (2018). SEER Cancer Statistics Review, 1975-2015, National Cancer Institute. Bethesda, MD, USA. https://seer.cancer.gov/csr/ 1975_2015/, based on November 2017 SEER data submission, posted to the SEER web site, April 2018.

Ono M., Ogilvie J.M., Wilson J.S., Green, H.J., Chambers, S.K., Ownworth, T., \& Shum, D.H.K. (2015). A meta-analysis of cognitive impairment and decline associated with adjuvant chemotherapy in women with breast cancer. Frontiers in Oncology 5: 59 doi: 10.3389/fonc.2015.00059

Pomykala, K.L., de Ruiter, M.B., Deprez, S., McDonald, B.C., \& Silverman, D.H. (2013). Integrating imaging findings in evaluating the post-chemotherapy brain. Brain Imaging and Behavior, 7 , 436-452.

Root, J.C., Andreotti, C., Tsu, L., Ellmore, T.M., \& Ahles, T.A. (2016). Learning and memory performance in breast cancer survivors 2 to 6 years post-treatment: the role of encoding versus forgetting. Journal of Cancer Survivorship: Research and Practice, 10, 593-599.

Schagen, S.B., Klein, M., Reijneveld, J.C., Brain, E., Deprez, S., Joly, F., Scherwath, A., Schrauwen, W., \& Wefel, J.S. (2014). Monitoring and optimising cognitive function in cancer patients: present knowledge and future directions. EJC Supplements, 12, $29-40$.

Schagen, S.B., Muller, M.J., Boogerd, W., Mellenbergh, G.J., \& van Dam, F.S. (2006). Change in cognitive function after chemotherapy: a prospective longitudinal study in breast cancer patients. Journal of the National Cancer Institute, 98, 1742-1745.

Scherling, C., Collins, B., MacKenzie, J., Bielajew, C., Smith, A.M. (2011). Pre-chemotherapy differences in working memory in breast cancer patients compared to controls: an fMRI study. Frontiers in Human Neuroscience, 5, 1-21.

Schilder, C.M., Seynaeve, C., Linn, S.C., Boogerd, W., Gundy, C.M., Beex, L.V., van Dam, F.S., \& Schagen, S.B. (2010). The impact of different definitions and reference groups on the prevalence of cognitive impairment: a study in postmenopausal breast cancer patients before the start of adjuvant systemic therapy. Psycho-Oncology, 19, 415-422.

Schmand, B., Lindeboom, J., \& Harskamp, F. (1992). De Nederlandse leestest voor volwassenen (the dutch adult reading test). Lisse, The Netherlands: Swets \& Zeitlinger.

Seigers, R., Schagen, S.B., Van Tellingen, O., \& Dietrich, J. (2013). Chemotherapy-related cognitive dysfunction: current animal studies and future directions. Brain Imaging and Behavior, 7, 453-459.

Shilling, V., Jenkins, V., \& Trapala, I.S. (2006). The (mis) classification of chemo-fog-methodological inconsistencies in the investigation of cognitive impairment after chemotherapy. Breast Cancer Research and Treatment, 95, 125-129.

Tager, F.A., McKinley, P.S., Schnabel, F.R., El-Tamer, M., Cheung, Y.K., Fang, Y., Golden, C.R., Frosch, M.E., Habif, U., Mulligan, M.M., Chen, I.S., \& Hershman, D.L. (2010). The cognitive effects of chemotherapy in post-menopausal breast cancer patients: a controlled longitudinal study. Breast Cancer Research and Treatment, 123, 25-34.

Vearncombe, K.J. \& Pachana, N.A. (2009). Is cognitive functioning detrimentally affected after early, induced menopause? Menopause (New York, N.Y.), 16, 188-198.

Wefel, J.S., Saleeba, A.K., Buzdar, A.U., \& Meyers, C.A. (2010). Acute and late onset cognitive dysfunction associated with chemotherapy in women with breast cancer. Cancer, 116, 3348-3356.

Wefel, J.S. \& Schagen, S.B. (2012). Chemotherapy-related cognitive dysfunction. Current Neurology and Neuroscience Reports, 12, 267-275.

Wohldmann, E.L., Healy, A.F., \& Bourne, L.E. (2008). A mental practice superiority effect: less retroactive interference and more transfer than physical practice. Journal of Experimental Psychology. Learning, Memory, and Cognition, 34, 823-833.

Yao, C., Bernstein, L.J., \& Rich, J.B. (2017). Executive functioning impairment in women treated with chemotherapy for breast cancer: a systematic review. Breast Cancer Research and Treatment, 166, 15-28. 